\title{
EL WIKI DE LA ORIENTACIÓN Y EL ASESORAMIENTO VOCACIONAL
}

\author{
THE GUIDANCE AND VOCATIONAL COUNSELLING WIKI
}

\author{
Jesús Sanz Esbri*, José Manuel Gil Beltrán** y Andrés Marzal Varóo** \\ Universitat Jaume I de Castellón
}

\section{RESUMEN}

Las redes telemáticas permiten a los orientadores compartir ideas y una gran cantidad de información sobre temas relevantes para su profesión. Por otra parte, la ingente labor que realizan en sus centros de trabajo da lugar a numerosos documentos, materiales o recursos de interés para otros orientadores y profesionales diversos. Estos materiales son infrautilizados por la falta de canales de comunicación e intercambio entre los mismos profesionales. En este sentido, EL WIKI DE LA ORIENTACIÓN Y EL ASESORAMIENTO VOCACIONAL se configura como un entorno colaborativo de hipertexto, accesible a través de Internet, en el que todos los orientadores y asesores vocacionales pueden compartir ideas, encontrar contenidos y recursos útiles para su labor, y en el que, ellos mismos, pueden colaborar activamente, añadiendo y editando los contenidos, actuales y futuros.

Palabras clave: asesoramiento vocacional, orientación vocacional, TIC, wiki.

\section{ABSTRACT}

Telematic networks allow guidance counsellors to share ideas and large amounts of information on issues relevant to their professions. The enormous amount of work carried out in their workplaces gives rise to many documents, materials or resources of great interest to other guidance counsellors

* Jesus Sanz Esbri es orientador escolar en el Colegio Ntra. Sra. de la Consolación de Castellón. Doctor en psicología. Actualmente, colabora con los Departamentos de Psicología y de Informática de la Universitat Jaume I de Castellón en el diseño y elaboración de herramientas aplicadas al asesoramiento vocacional. E-mail: 5195jse@cop.es

** Juan Manuel Gil Beltrán es profesor titular de Psicología evolutiva y de la educación en la Universitat Jaume I de Castellón. Sus líneas de investigación versan sobre el asesoramiento vocacional en las etapas educativas de secundaria y universidad. Actualmente, su interés se centra en la adecuación del proceso de asesoramiento vocacional y toma de decisiones a los nuevos retos europeos.

*** Andrés Marzal Varó es profesor titular de Lenguajes y sistemas informáticos en la Universitat Jaume I de Castellón. Sus líneas de investigación giran en torno a la aplicación de las nuevas tecnologías al proceso de enseñanza-aprendizaje. Actualmente, colabora con el Departamento de Psicología en el diseño y elaboración de herramientas aplicadas al asesoramiento vocacional. 
and a range of other professionals. However, because of the lack of communication and exchange channels among professionals, these materials are underused. The Guidance and Vocational Counselling Wiki fills this gap in the form of a collaborative hypertext environment, accessible through the Internet, in which all guidance counsellors and vocational advisers can share ideas, find useful material and resources for their work, and actively participate by adding and editing present and future content.

Keywords: vocational counselling, school guidance, ICT, WIKI.

\section{Introducción}

Es indudable que los frutos del trabajo en colaboración son mayores que los conseguidos a partir del que se elabora individualmente, y esta cuestión es especialmente importante cuando se trata de realizar tareas dentro de una empresa u organización. Gracias al software para el trabajo en grupo es factible la mejora del rendimiento en general de todo el proceso productivo y su mayor aportación es hacer posible que diferentes personas puedan trabajar de forma compartida con una misma información y cooperar estrechamente en el desarrollo de proyectos. Este tipo de herramientas está lo suficientemente difundida en un entorno no tan amplio como el web, sobre todo en las Intranets corporativas (Castillo, 1999). Dentro de este contexto, las funciones básicas que se consiguen llevar a cabo con su utilización son, según Hills (1997), las siguientes:

- Ayuda a que dos personas, o más, trabajen juntas.

- Permite compartir conocimientos y experiencias.

- Automatiza sus actividades.

- Ayuda a crear una memoria de la organización.

- Aúna geografía y tiempo.

Es una herramienta poderosa que permite compartir toda clase de conocimiento relativo a una organización y facilita el movimiento y control de la información que se manipula constantemente.

Por otra parte, cada día es más frecuente que profesionales pertenecientes a diferentes universidades, instituciones o empresas lleven a cabo investigaciones aparte de las funciones normales que realizan en sus lugares habituales de trabajo. Aunque la red aporta una serie de instrumentos para esta colaboración, se hacía necesaria una herramienta capaz de crear en el web un espacio compartido en donde desarrollar y mantener almacenados proyectos comunes de forma totalmente fiable y segura.

Hasta el momento, el sistema más utilizado para manipular documentos, de forma más o menos paralela, ha sido el correo electrónico y, sobre todo, las listas de distribución. El envío de ficheros a partir de ellos es constante y junto con el servicio de news constituyen básicamente la oferta de Internet para el trabajo en grupo. Hay que tener en cuenta, sin embargo, que no se hace en un área específica para ello, sino que simplemente hay un movimiento de información de un lugar a otro de la red y esta situación plantea el problema de que las diferentes versiones que se crean progresivamente de un mismo fichero no están controladas en un único espacio ni accesibles en cualquier momento a todos los posibles participantes. 
Otra herramienta, como lo es el chat, no ha contado hasta ahora con la aceptación por parte de la comunidad científica y profesional. La razón, según Coma del Corral (1998), es que ha estado desde su creación más enfocado al entretenimiento que al trabajo técnico propiamente dicho. No obstante, no puede negarse que es el método más eficaz que Internet pone al alcance de sus usuarios para la comunicación a tiempo real sin que esto conlleve un gasto tecnológico alto. No es menos obvio que este sistema no es el más apropiado para difundir una información relevante fruto de una investigación rigurosa. De cualquier forma, tampoco debe despreciarse su uso simplemente por las implicaciones negativas que conlleva y es necesario saber encontrar aplicaciones dentro de un contexto científico que aproveche sus posibilidades reales de interacción.

Con la proliferación de las comunidades virtuales de usuarios (CVU) de carácter profesional, la necesidad de dar soporte a ese trabajo interinstitucional es cada vez más importante. La característica principal de cualquiera de estas CVU debe ser la de ofrecer las herramientas adecuadas para que se lleve a cabo una labor que aspira, día a día, a ser cada vez más colaborativa $\mathrm{y}$, sobre todo, que fomente la participación de un mayor número de personas.

En el ámbito del web este software no se encuentra tan perfeccionado como en el caso de las redes corporativas.

En orientación, concretamente, se han desarrollado webs de recursos y materiales de interés para el orientador y el asesor vocacional. Sin embargo, no parece existir un entorno web "verdaderamente" colaborativo, un sistema en el que todos los participantes, todos los orientadores que así lo deseen, puedan: ver contenidos e informaciones de interés para su trabajo, debatir ideas y temas diversos, "bajarse" documentos y materiales, acceder a recursos y servicios, realizar sus propias contribuciones, editando y modificando los mismos contenidos de la web, añadiendo sus materiales y recursos, etc.

Con esta finalidad nace el WIKI DE LA ORIENTACIÓN Y EL ASESORAMIENTO VOCACIONAL.

\section{Los "Wikis", el fenómeno del "Software libre" y los entornos de desarrollo de aplicaciones Web}

Un Wiki es una web colaborativa, que intenta escapar de los convencionalismos de otro tipo de portales/sites y utiliza un paradigma poco común: la posibilidad de permitir a todo el mundo editar las páginas web que contiene, o añadir otras nuevas.

El primer WikiWikiWeb fue creado por Ward Cunningham, quien inventó y dio nombre al concepto Wiki, (el término WikiWiki significa "rápido" en la lengua hawaiana) y produjo la primera implementación de un servidor WikiWiki.

Dada la rapidez con la que se actualizan los contenidos, la palabra "wiki" adopta todo su sentido. El documento de hipertexto colaborativo resultante, denominado también wiki o WikiWikiWeb, se produce típicamente por una comunidad de usuarios.

Como principales ventajas podemos señalar que: 
- Una persona A puede escribir sobre un tema determinado, aportando información y referencias. En un Wiki, otra persona B puede corregir o ampliar aquello que puso A, con el propósito de mejorarlo.

- Si se consigue un volumen de contribuidores adecuados, un Wiki es probablemente la mejor manera de compartir conocimientos: es autoalimentado.

- Un Wiki es extensible a discreción del usuario. Esto da mucha flexibilidad a la hora de estructurar la web por temas, y no mezclar cosas. Además, fomenta de manera natural la inclusión de relaciones entre estos temas.

Problemas y desventajas:

- La buena estructura del Wiki debe mantenerse basándose en el criterio de los usuarios. En un Wiki cualquiera puede modificar una página (de la cual se hace un diff - comparación entre dos archivos - de manera inmediata), dejando abierta la posibilidad a la incorporación de contenidos prohibidos o acciones malintencionadas.

Algo positivo y rescatable es que cuando se consigue una determinada masa crítica, el problema del ingreso de contenidos dañinos desaparece, o mejor dicho se soluciona rápidamente. Los Wikis generalmente funcionan correctamente porque si hay alguna introducción con mala fe por parte de un usuario, es muy probable que aparezca otro y deshaga estos cambios (en un Wiki se guardan todas las versiones de una página, para hacer esto posible). Si no se dispone de esa masa crítica de usuarios entonces el Wiki es más vulnerable y depende, en gran medida, de un administrador que supervise estas cosas y las arregle.

El concepto de los wikis se basa en "hermanar" múltiples sitios de similares características, con la particularidad de que el contenido se alimenta de los aportes de los usuarios, que pueden alterar y modificar contribuciones ajenas.

En general es utilizado por usuarios que están agrupados por temas de afinidad o intereses comunes. Estos usuarios tienen un espíritu participativo que es el que permite el crecimiento y actividad de cada Wiki. Los mismos deben ser responsables de sus acciones en el Wiki, para así permitir un desarrollo ordenado de los contenidos.

En otro orden de cosas, el movimiento del "software libre" (traducción del inglés free software) o del "código abierto" (en inglés, open source) se plantea como meta que el código fuente de todo programa esté disponible para cualquier usuario, de modo que éste pueda modificarlo para corregir errores o mejorar sus prestaciones. Generalmente se impone una condición: que si se distribuye un programa modificado por el usuario, los cambios también se hagan públicos y se distribuyan bajo el mismo modelo de "software libre".

El desarrollo de Internet está fuertemente vinculado al "software libre". No sólo es la herramienta más usada para comunicar entre sí a los usuarios; es también un campo fértil para la creación de nuevas aplicaciones.

Uno de estos campos es el de los lenguajes y entornos para facilitar el desarrollo de nuevas "aplicaciones web". Se trata, pues, de "meta-aplicaciones" que simplifican notablemente el desarrollo de programas capaces de interactuar con usuarios ubicados en cualquier punto de la red y que usan un simple navegador como interfaz.

Entre los sistemas de desarrollo y soporte de aplicaciones web más destacables tenemos PHP y Zope, ambos "libres". El más popular es el primero. Si bien no es tan potente como el segundo, presenta una curva de aprendizaje mucho más moderada y es posible pasar del nivel básico al desarrollo de aplicaciones de complejidad moderada en poco tiempo. 
Hay que indicar que PHP es sólo el lenguaje de programación y un módulo para su interpretación. Una solución completa requiere utilizar un ordenador con sistema operativo, un servidor de páginas web y, generalmente, una base de datos. La configuración más corriente utiliza Linux como sistema operativo, Apache como servidor y MySQL como base de datos. La integración de estas cuatro herramientas (sin excluir Python y Perl como sustitutos de PHP) se conoce como LAMP (http://www.lamp.org).

Moodle es un sistema escrito en PHP que aglutina numerosos módulos para el diseño de cursos virtuales, aunque también resulta útil para la creación de portales de grupos de interés común. Entre los módulos y funcionalidades de Moodle podemos destacar los relacionados con los foros, repositorio de documentos, wikis y gestión de colecciones de enlaces de interés.

La web de nuestro WIKI esta implementada con Moodle y comparte, por tanto, la filosofía del "software libre", por lo que puede ser utilizada, distribuida y modificada con las únicas restricciones de que debe indicarse siempre la fuente de los programas y de que sus modificaciones sean, también, "software libre".

\section{El Wiki de la orientación y el asesoramiento vocacional}

\section{Utilidad y finalidad}

La utilización de las tecnologías de la información y comunicación en manos de los orientadores abre nuevas expectativas para su desarrollo profesional. A través de las redes los orientadores pueden compartir una gran cantidad de información sobre nuevos recursos, temas relevantes para su profesión y nuevas ideas. Las redes posibilitan el acceso a un enorme conjunto de personas que están trabajando en el mismo tema, locales e internacionales y obtener ayuda desde una amplia variedad de fuentes, todo ello desde un enfoque colaborativo del propio desarrollo profesional (Harasim et al., 1995).

En este sentido, como apunta Salinas (1998), las tecnologías de la información proporcionan la oportunidad de acceder a una diversidad de servicios como pueden ser:

a) Obtención de servicios especializados de información que existen en cada campo académico y profesional y que suelen ser accesibles para los miembros de la disciplina o de la profesión.

b) Intercambio de nuevos conocimientos surgidos tanto de la investigación básica y aplicada como de la práctica profesional a través de revistas electrónicas, conferencias electrónicas y listas de discusión.

c) Colaboración para mejorar las aptitudes y resolver problemas. Este tipo de colaboración es requerida más que el simple intercambio de información. Intercambio de ideas, compartir experiencias y discutir soluciones a las dificultades que se dan entre profesionales, por ejemplo, pueden dar lugar, a través de redes a grupos de trabajo mucho más amplios y diversos.

d) Colaboración para crear nuevo conocimiento. Distintas personas trabajan juntos durante largos periodos para lograr metas compartidas: un grupo de profesores desarrollando materiales curriculares o escribiendo un artículo en común, la realización de investigaciones y proyectos de innovación por equipos de profesores de distintos 
centros, el intercambio de borradores y planes de trabajo... constituyen típicos ejemplos de esta categoría.

En el caso de los profesionales de la orientación, debemos remarcar que la ingente labor que realizan da lugar, muchas veces, a numerosos documentos, materiales o recursos que serían muy interesantes para otros orientadores y profesionales diversos. Estos materiales son infrautilizados por la falta de canales de comunicación e intercambio entre los mismos profesionales.

Por otra parte, esa comunicación, esa interacción entre los profesionales de la orientación permitiría comentar y debatir temas de interés (foros), crear nuevos materiales en colaboración, actualizarse permanentemente, ...

Este es el principal objetivo de nuestro WIKI DE LA ORIENTACIÓN Y EL ASESORAMIENTO VOCACIONAL, su principal deseo: convertirse en un entorno para compartir rápidamente ideas, crear bases de conocimiento sobre la orientación y el asesoramiento vocacional, interactuar con una comunidad de profesionales interesados en el campo de la orientación, etc. La idea fundamental del Wiki consiste en permitir que cualquier miembro de la comunidad altere el contenido del Wiki. En el modo edición, no sólo se le permite añadir contenido mediante comentarios (que es lo habitual en los foros de noticias y weblogs al uso), sino que se permite modificar contenidos existentes y crear nuevas páginas. Los usuarios pueden participar añadiendo información, actualizando la que crean obsoleta o corrigiendo aquella que consideren errónea, contribuyendo, de esta manera, a crear un almacén de información útil.

En un primer momento, el profesional de la orientación que acceda a la web se encontrará con una serie de apartados en los que estarán distribuidos los contenidos, materiales y recursos. Es nuestra propuesta inicial. A partir de ella, los orientadores que así lo deseen podrán modificar los aspectos contenidos en cada uno de estos apartados, implementar sus propias aportaciones, e, incluso, editar el nombre de los propios apartados, borrando y añadiendo nuevos temas de interés.

\section{Tabla de contenidos inicial}

- Formación permanente.

- Nuevas Tecnologías.

- Materiales de Orientación.

- Bibliografía.

- Congresos.

- Tesis Doctorales.

- Asociaciones Nacionales.

- Asociaciones Internacionales.

- Enlaces importantes.

- Líneas de trabajo/investigación.

- Elaboración de materiales.

- ¿Quién me puede decir algo sobre...? 
Contenido. Mapa de navegación y estructura de la web

Podemos acceder al WIKI DE LA ORIENTACIÓN Y EL ASESORAMIENTO VOCACIONAL a través de la página web del SITIO WEB DE LA ORIENTACIÓN Y EL ASESORAMIENTO VOCACIONAL y su COMUNIDAD DE ORIENTADORES, como invitados o identificándonos con nuestra contraseña en la página http://imp.act.uji.es/moodle/ login/index.php.

La COMUNIDAD DE ORIENTADORES, a la que hacemos referencia en estas líneas, forma parte de un tipo específico de comunidad virtual: el constituido por el intercambio entre profesionales. La posibilidad y el desafío de compartir miradas diferentes acerca de problemáticas comunes, crea un espacio privilegiado de aprendizaje de relaciones que suelen configurarse como exigencias más o menos habituales en la práctica profesional. En la búsqueda misma de información, los profesionales de la orientación pueden necesitar el mismo tipo de servicios o herramientas y, sobre todo, y más importante, pueden encontrar una vía de enriquecimiento profesional a través del intercambio de conocimiento, materiales o recursos. La COMUNIDAD DE ORIENTADORES recoge algunos de estos servicios e integra toda una serie de instrumentos desarrollados por la investigación - entre los que se encuentra el WIKI DE LA ORIENTACIÓN Y EL ASESORAMIENTO VOCACIONAL - (Sanz, 2005), en un punto de encuentro, un portal web de acceso a recursos, información, materiales, herramientas y servicios de interés, con la idea de que los profesionales de la orientación y el asesoramiento vocacional compartan e intercambien información a la vez que trabajan en proyectos comunes.

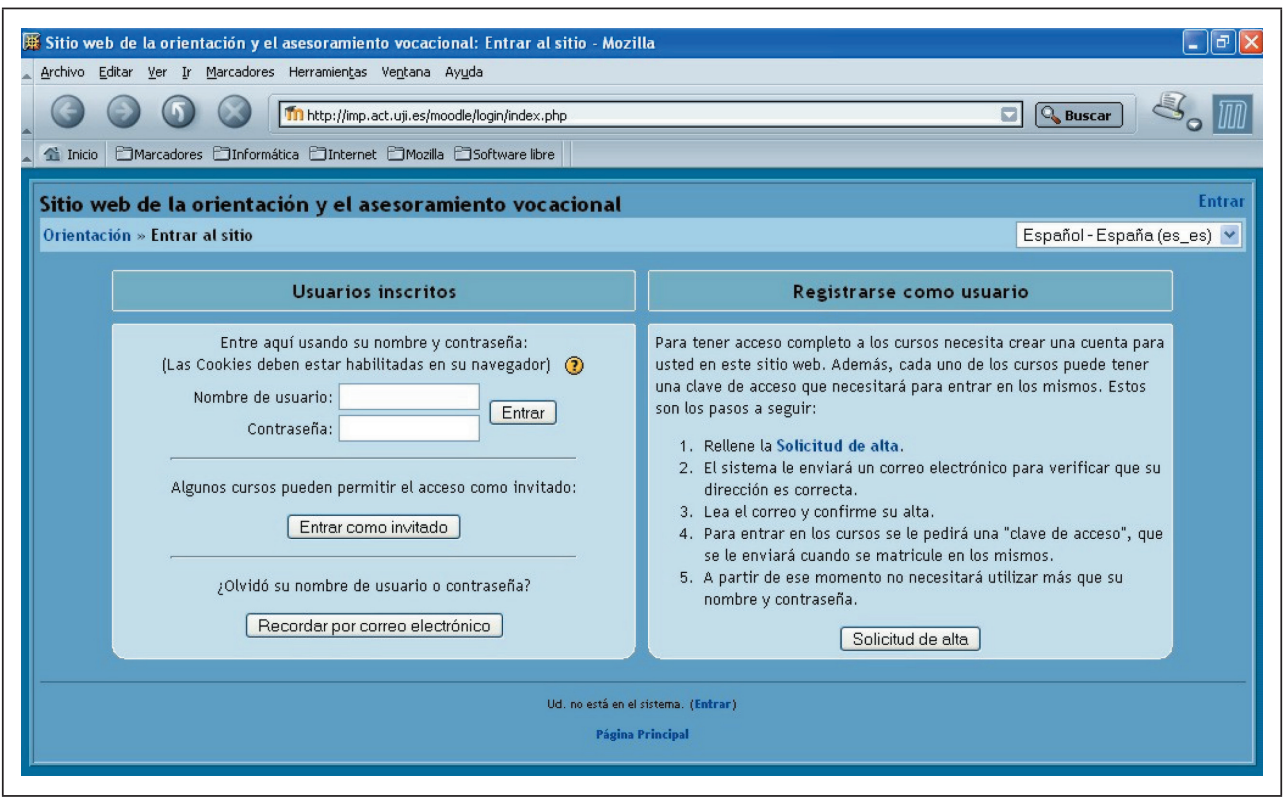

FIGURA 1.

Página de Entrada al Sitio Web de la Orientación y el Asesoramiento Vocacional. 
Como invitados, podremos ver todos los contenidos de la web y tener acceso a muchos de los materiales y recursos disponibles en la misma, pero no podremos modificar/editar sus contenidos. Para tener un acceso completo a la web y contar con la posibilidad de participar activamente en la COMUNIDAD, aportando nuevos materiales o informaciones y modificando los contenidos existentes, deberemos darnos de alta en el sistema. Para ello, rellenaremos la solicitud de alta disponible en la pantalla de Entrada.

Tanto si accedemos como miembros registrados o como usuarios invitados, tras la pantalla inicial de identificación, entraremos en una nueva pantalla, igual en ambos casos, en la que nos encontraremos con diversa información (Novedades o foros/temas de debate abiertos, Usuarios conectados, ...) y con la posibilidad de acceder a los diferentes cursos o comunidades implementados/as en el sitio web - actualmente, a nuestra COMUNIDAD DE ORIENTADORES-.

La pantalla inicial de la COMUNIDAD DE ORIENTADORES presentará un aspecto similar para el usuario invitado y el miembro de pleno derecho, pero el usuario registrado contará con algunas opciones más como las de editar su propios datos o información personal, cambiar su contraseña y darse de baja como usuario registrado.

Sólo con pulsar con nuestro ratón sobre el enlace del WIKI en el menú Actividades sociales (ver figura 2), accederemos a su página web inicial. En ella, tanto si accedemos como invitados como si lo hacemos identificándonos con nuestra contraseña, encontraremos en la parte superior algunos apartados propios de la "COMUNIDAD DE ORIENTADORES", como el desplegable "Saltar a..." y los enlaces a la jerarquía web de los que depende la página.

Contaremos, también en uno y otro caso, con la posibilidad de buscar cualquier texto dentro del sitio web del WIKI, introduciéndolo en el cuadro de texto "Buscar Wiki".

Pulsando sobre alguno de los resultados obtenidos, accederemos directamente al contenido relacionado. Ésta es una característica implementada en todo el sitio web: siempre, desde cualquier punto donde nos encontremos, podremos acceder directamente a otro con una simple pulsación de nuestro ratón sobre el enlace (texto de color azul) que nos interese. De igual forma, aparecerán enlaces directos a otros sitios web, direcciones de correo electrónico, documentos y materiales de interés no contenidos en el sitio web del WIKI, etc.

Por otra parte, también podremos seleccionar el lugar que nos interese visitar del sitio web a través de un desplegable con diferentes enlaces en "Elegir Enlaces Wiki" y acceder directamente al mismo, o habilitar una ayuda (útil, sobre todo, para el "modo edición") pulsando sobre el icono "?".

Sin embargo, si accedemos a la web como usuarios registrados, aparecerán algunas posibilidades con las que el usuario invitado no va a contar, en forma de pestañas de fácil acceso en la parte superior de la página (ver figura 3):

- Ver, para visionar la página web actual tal como la vería cualquier usuario, esté o no registrado.

- Edición, que habilita el "modo edición”, comentado en detalle más adelante.

- Enlaces, que relaciona las páginas con las que está vinculada la página visitada en ese momento.

- Historia, con el historial de modificaciones de la página. 


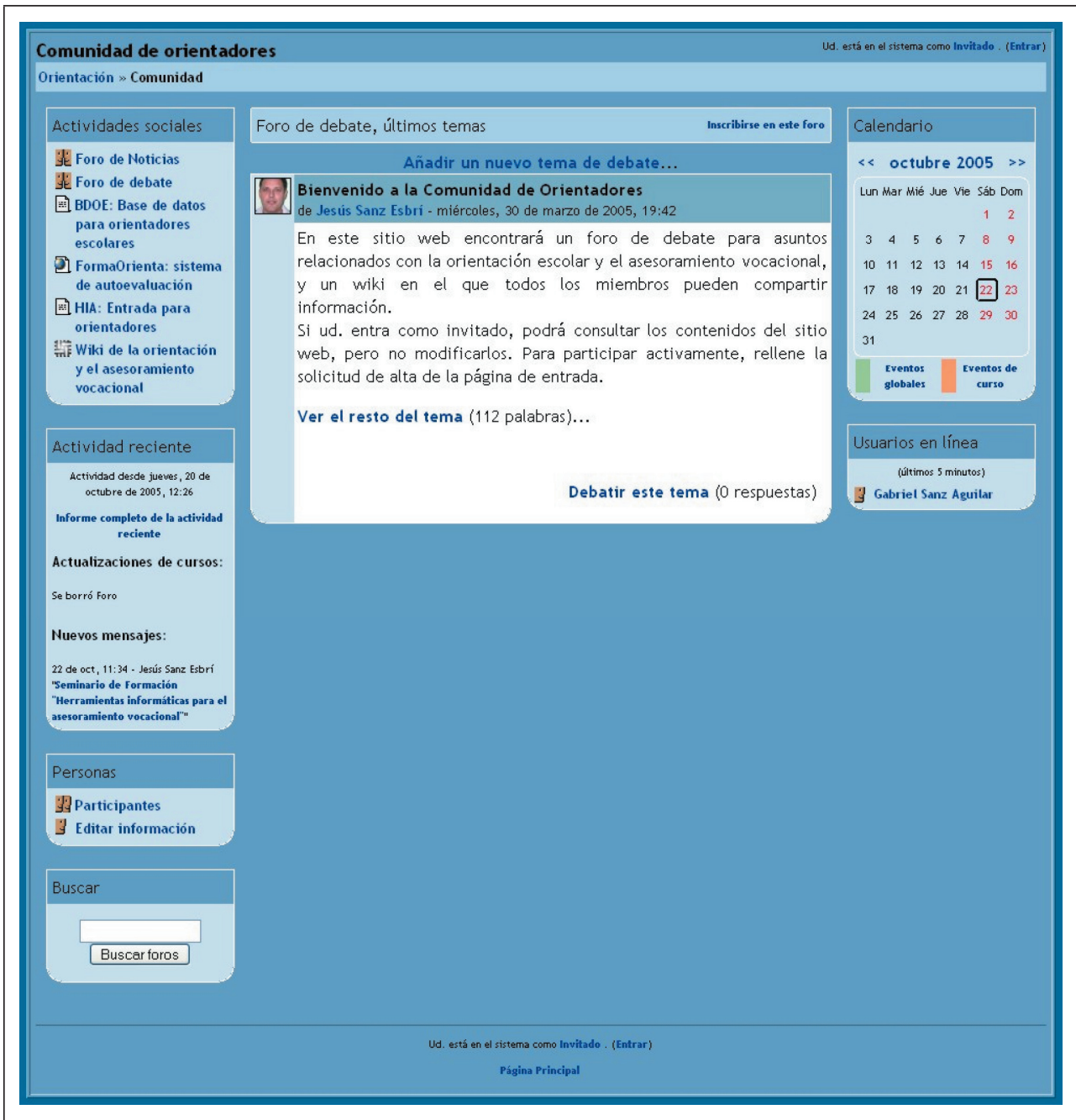

FIGURA 2.

Página inicial de la Comunidad de Orientadores (como usuario invitado).

- Anexos, que lista todos los documentos, materiales y recursos añadidos en forma de archivos anexos (attachments) a la página actual y permite añadir otros.

Junto a todo lo anterior, en la página inicial, tanto en su versión para usuarios registrados como para los invitados, aparecerá, después de una breve introducción al WIKI, una tabla con enlaces a los diferentes apartados contenidos en el sitio web (ver figura 3 ).

Es una propuesta inicial que, como antes señalábamos, puede y debe ser ampliada y mejorada con la colaboración de todos. 


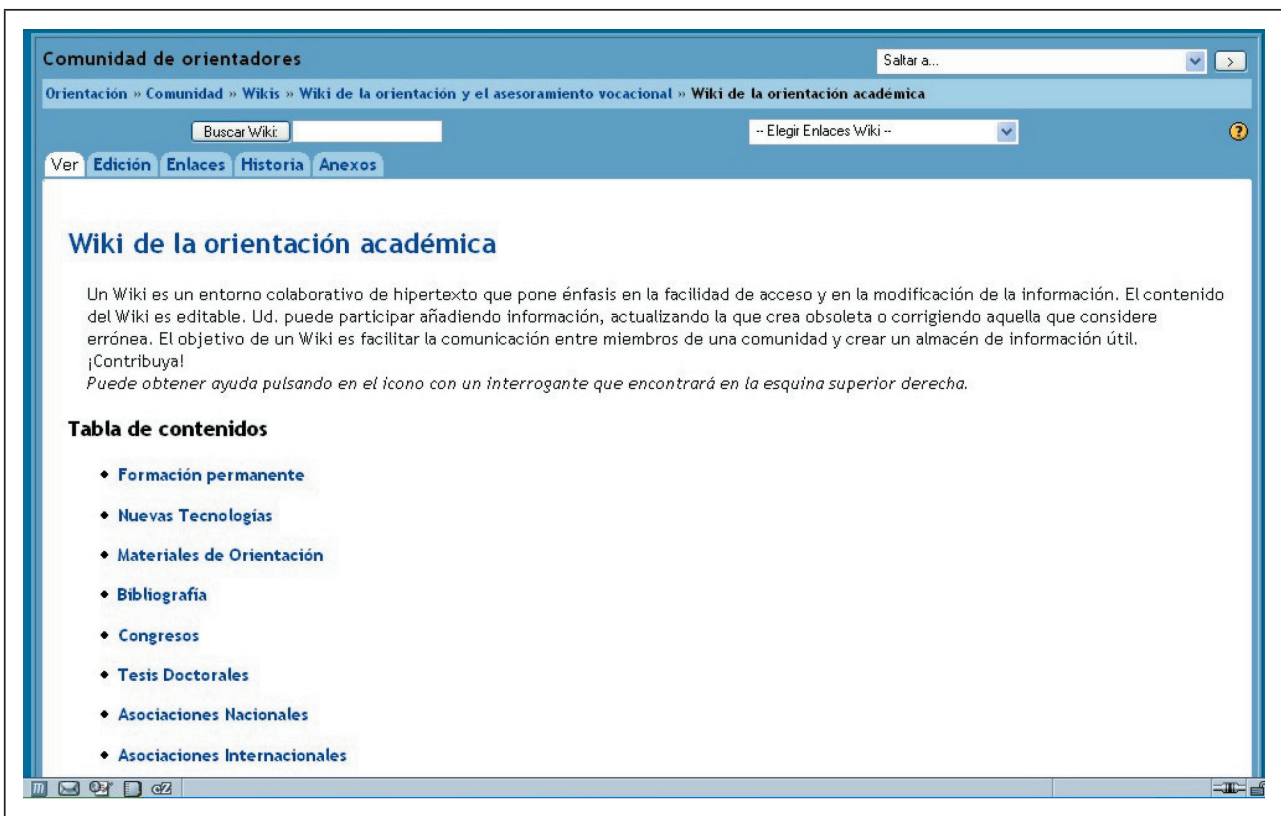

FIGURA 3.

Página inicial del WIKI (accediendo como usuario registrado).

Un ejemplo de lo que pretendemos lo forman los dos últimos apartados: "Elaboración de materiales" y "¿Quién me puede decir algo sobre...?". Estos configuran una llamada al trabajo colaborativo de los orientadores. De todos nosotros depende que esta propuesta sea aprovechada en su máxima expresión.

Como ya hemos señalado, contaremos con la posibilidad de editar y modificar los contenidos del sitio web, borrar información obsoleta, añadir nuevos materiales y recursos, etc. Para ello, el WIKI nos facilita una herramienta de edición que, a modo de procesador de textos, nos permitirá introducir cuantos cambios deseemos en el sitio web.

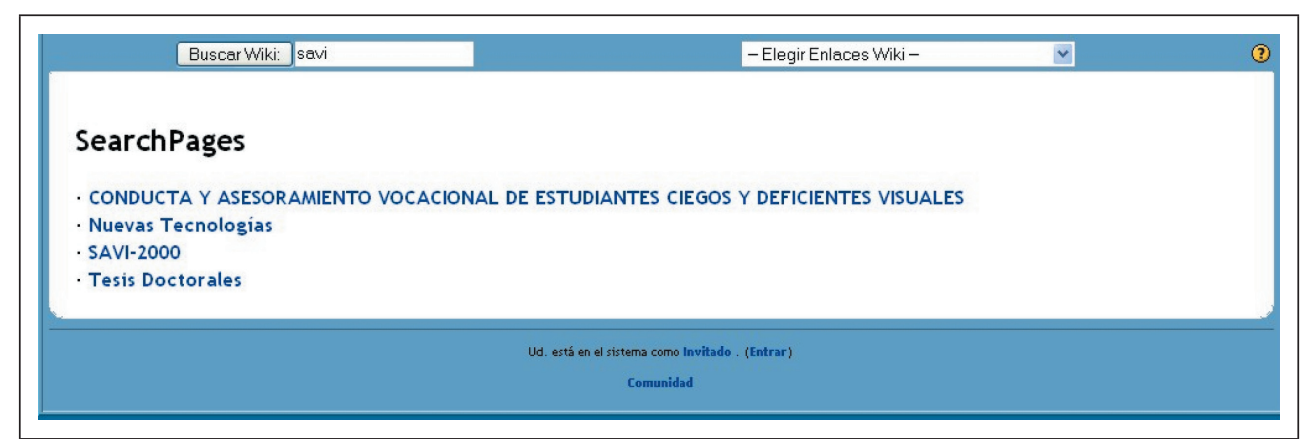

FIGURA 4.

Resultados para la búsqueda "savi". 


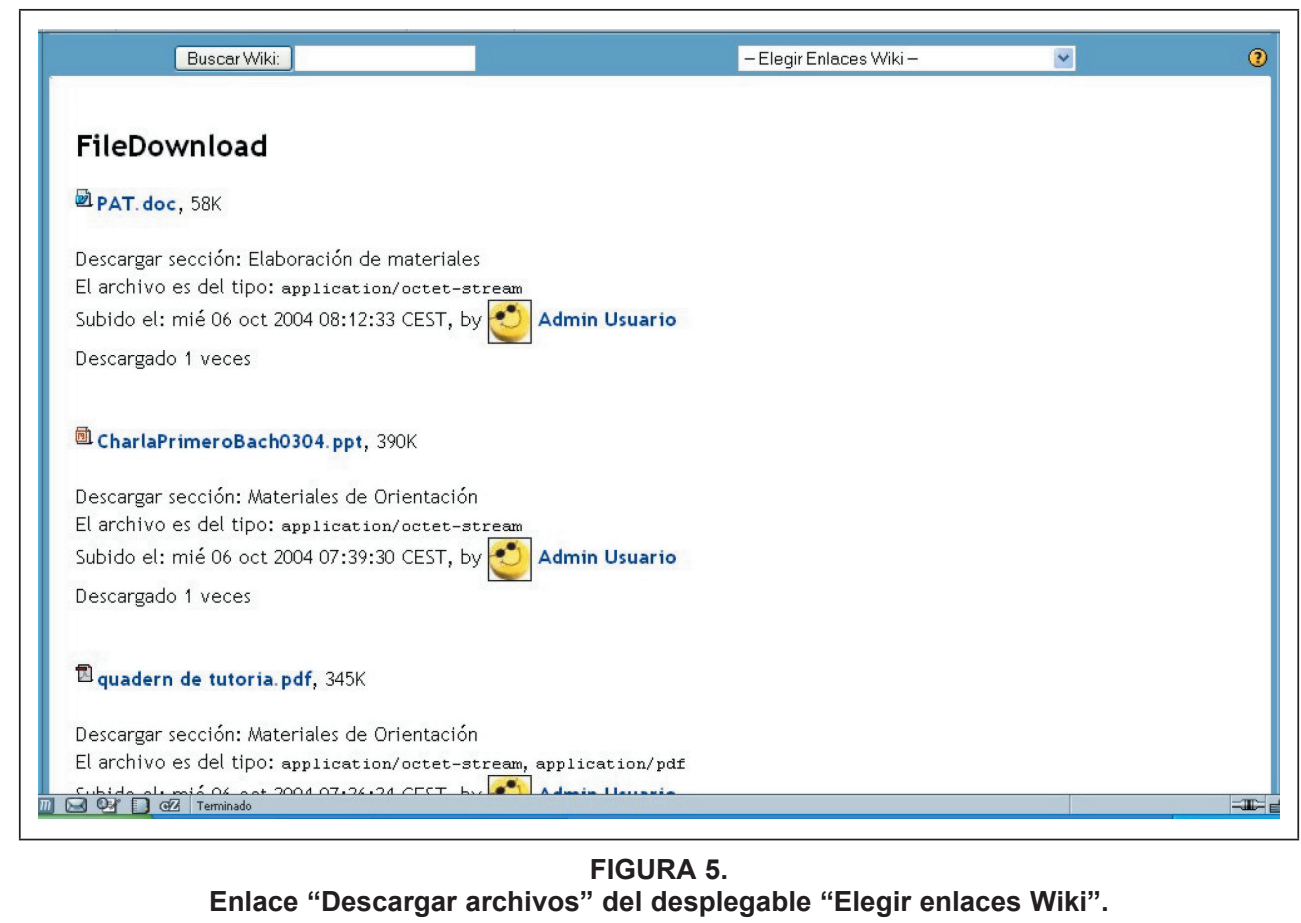

\section{Modo edición}

La herramienta de edición, como vemos en la figura 10, funciona como cualquier procesador de textos al uso. Deberemos, eso sí, atender a una serie de reglas, que conforman una manera diferente de escribir Wiki (y que podemos encontrar explicadas en la ayuda facilitada por el icono “?”). Así, por ejemplo, podremos crear una página dándole un nombre en el texto de una página ya existente. Una página wiki se nombra utilizando CamelCase (este término describe la apariencia de las PalabrasWiki — "WikiWords"-: varias palabras unidas sin espacios entre sí, mezclando mayúsculas y minúsculas — las letras mayúsculas y minúsculas se asemejan a las jorobas de un camello-. A este modo de establecer enlaces se denomina a menudo "BumpyText". En tanto que los puristas del lenguaje odian a Wiki por utilizar estos atentados idiomáticos, lo cierto es que son muy comunes en el mundo informático, y bien conocidos por la mayoría de los programadores) o bien poniendo el nombre entre corchetes ([ ]).

Por ejemplo:

- MyWikiPage (Camel Case).

- [My Wiki Page] (entre corchetes).

El nombre que se ha dado a una página wiki deberá tener un “?” después del mismo. Al hacer clic en "?", se entrará en el modo de edición de esa página. Si escribimos el texto y lo guardamos, tendremos una nueva página wiki. 


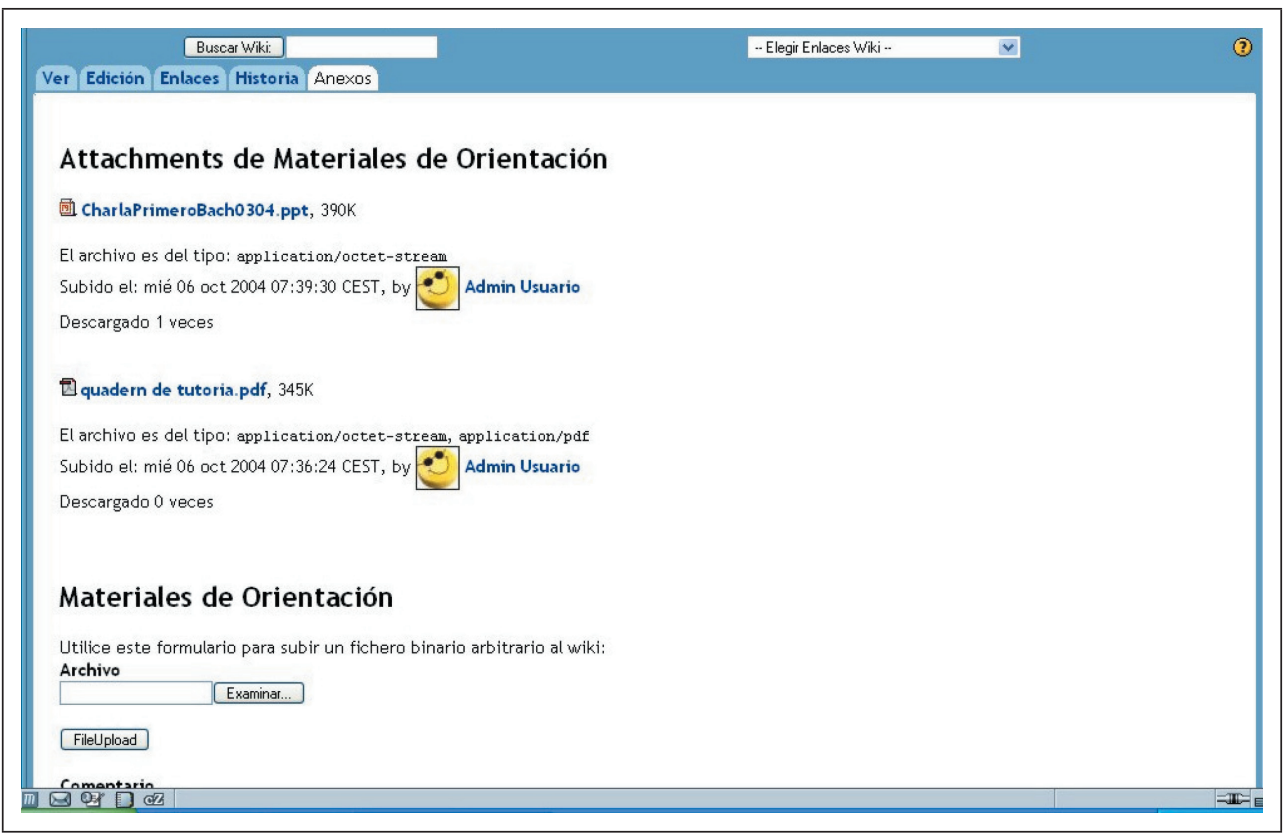

FIGURA 6.

"Anexos" del apartado "Materiales de Orientación".

\begin{tabular}{|l|}
\hline Tabla de contenidos \\
- Formación permanente. \\
- Nuevas Tecnologías. \\
- Materiales de Orientación. \\
- Bibliografía. \\
- Congresos. \\
- Tesis Doctorales. \\
- Asociaciones Nacionales. \\
- Asociaciones Internacionales. \\
- Enlaces importantes. \\
- Líneas de trabajo/investigación. \\
- Elaboración de materiales. \\
- ¿Quién me puede decir algo sobre...?
\end{tabular}

FIGURA 7.

Actual "Tabla de contenidos" del WIKI.

Por otra parte, con el formulario "Anexos" podremos también modificar el sitio web, añadiendo ficheros de todo tipo a la página que nos interese. Para ello, sólo tendremos que especificar la ruta, hacia el lugar en donde se encuentran ubicados, en el cuadro de texto 


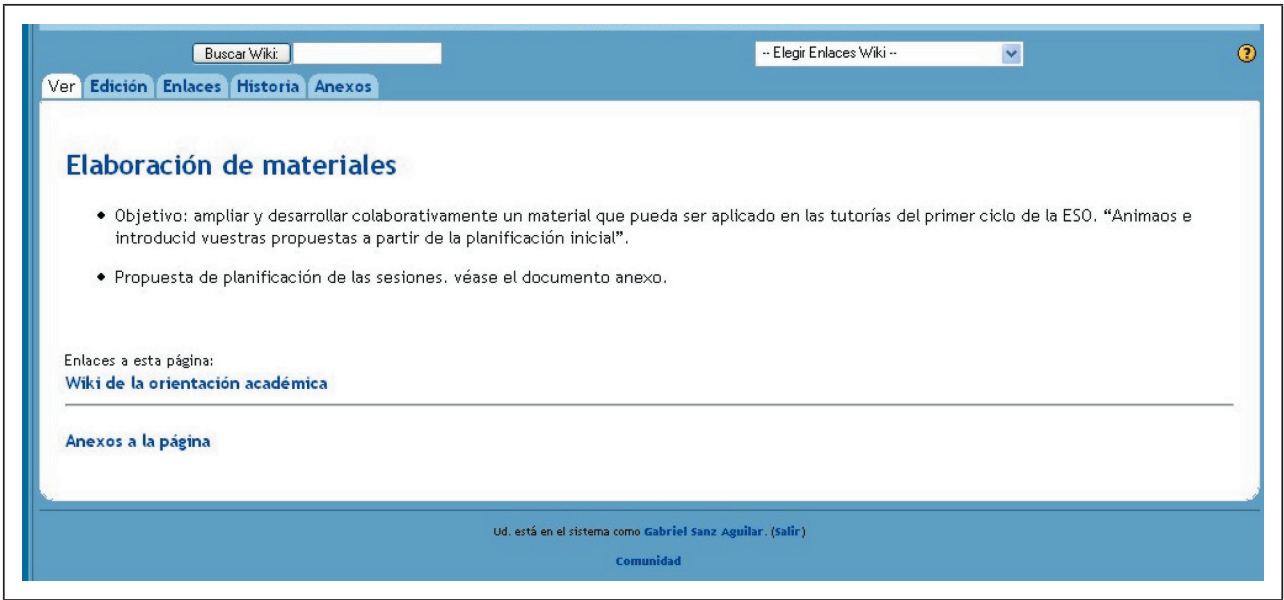

FIGURA 8.

Captura de pantalla del apartado "Elaboración de materiales".

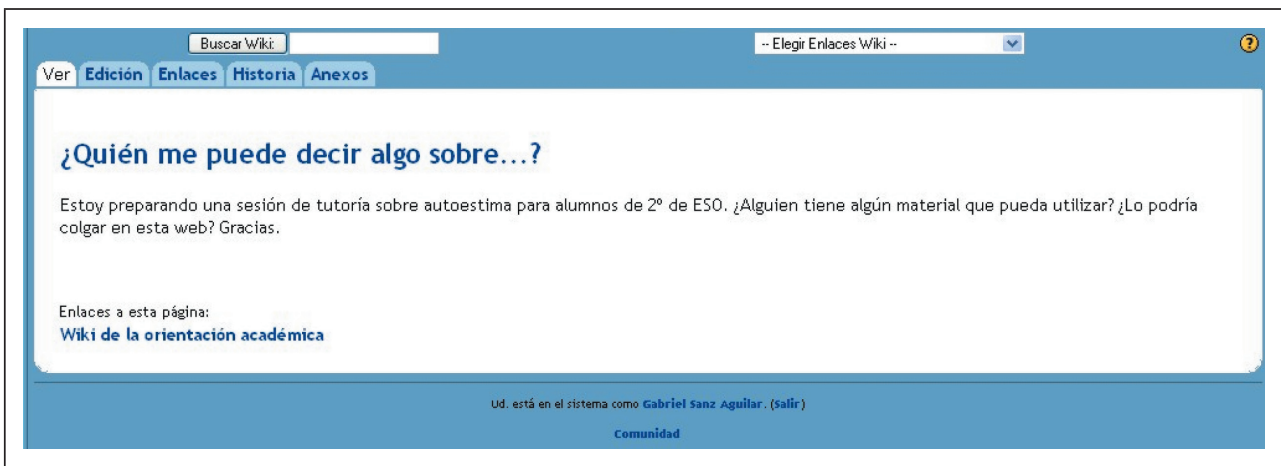

FIGURA 9.

Captura de pantalla del apartado “¿Quién me puede decir algo sobre...?”.

"Archivo", añadir un comentario, si lo deseamos, en el cuadro "Comentario" y "cargar" el archivo en la página de nuestra elección, seleccionándola del desplegable "Cargar en".

En último término, el Administrador del sitio se reserva el derecho de revisar y supervisar las modificaciones realizadas, pudiendo volver a alguna de las versiones anteriores del WIKI que siempre quedan almacenadas en el servidor.

\section{Valoración}

El WIKI DE LA ORIENTACIÓN Y EL ASESORAMIENTO VOCACIONAL, como hemos visto, es o trata de ser - eso va a depender de todos nosotros (los profesionales de la 


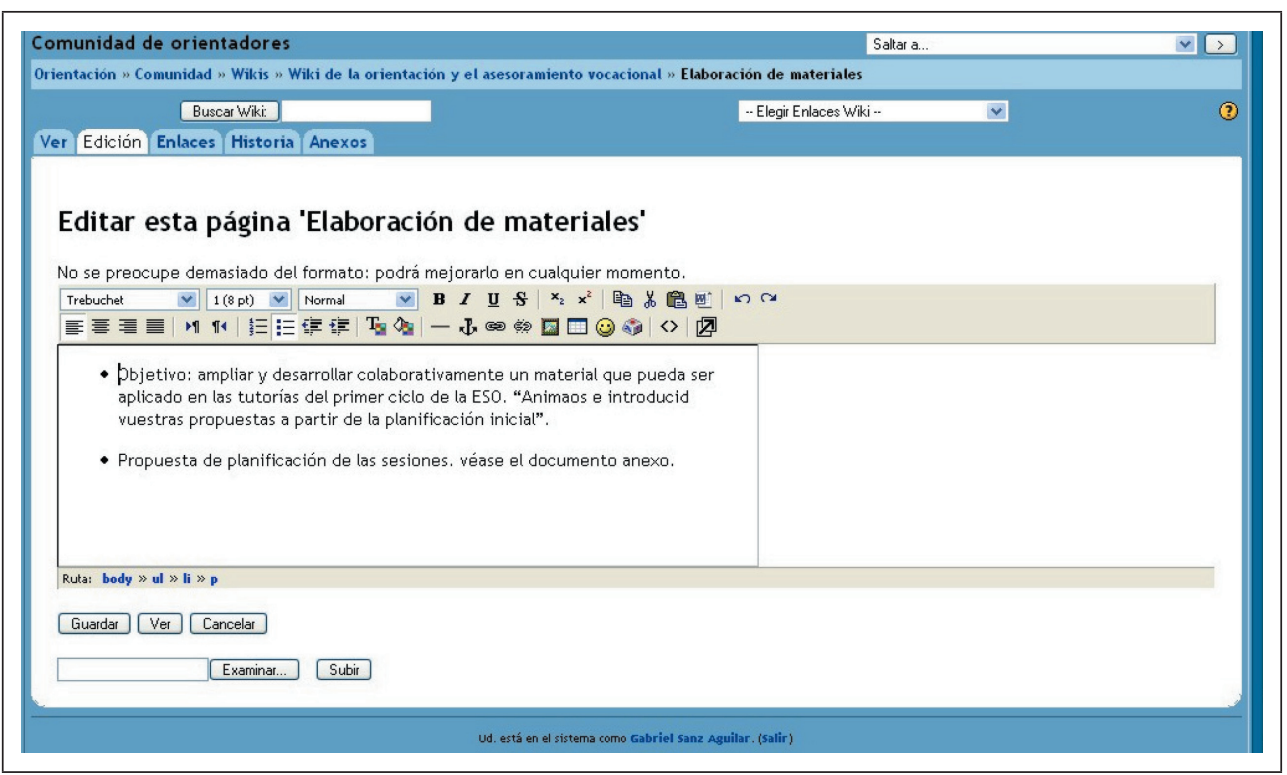

FIGURA 10

Pantalla de edición de la página "Elaboración de materiales".

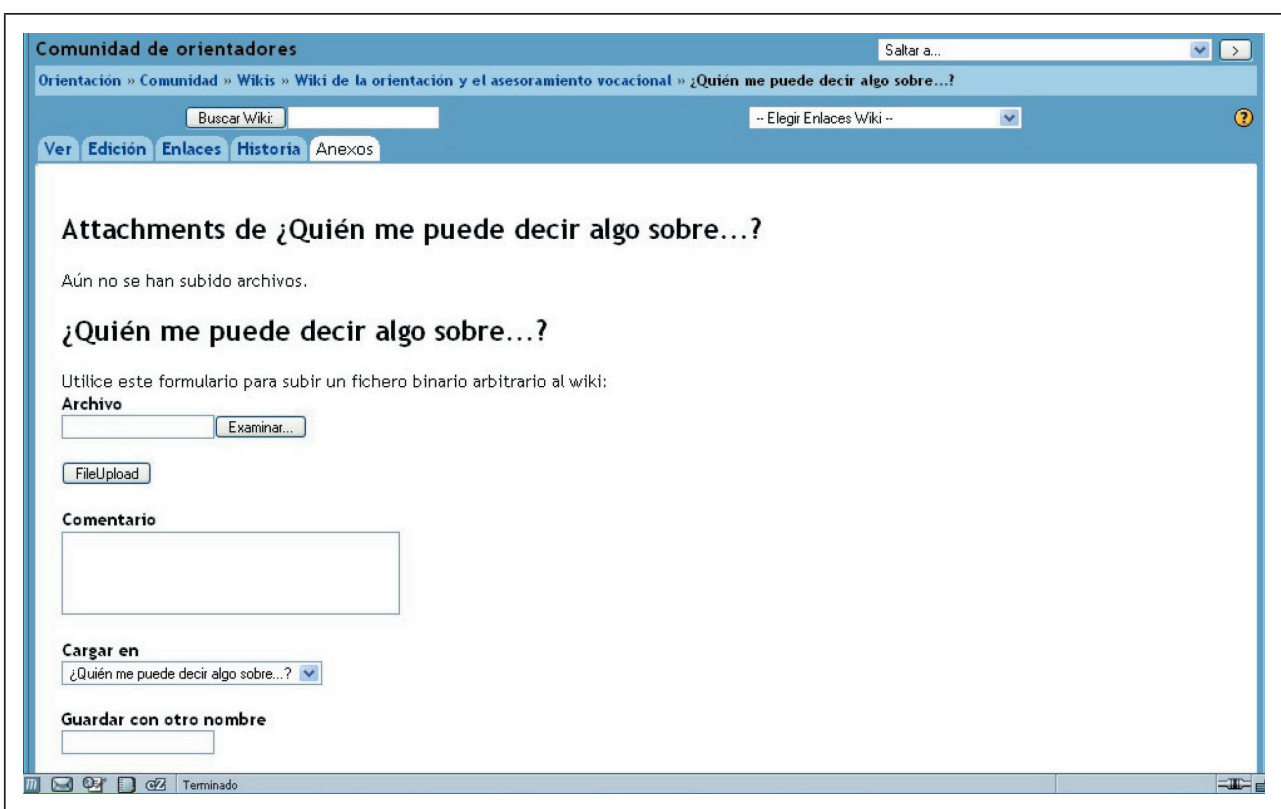

FIGURA 11.

Formulario “Anexos” del apartado “¿Quién me puede decir algo sobre...?”. 
orientación) - un entorno colaborativo en el que el trabajo de unos pocos sea aprovechado por muchos, realizando estos también sus propias contribuciones al sitio web de forma que éste se convierta en un centro de recursos y materiales, contenidos e información de interés para los profesionales de la orientación y el asesoramiento vocacional, en estado de actualización permanente y eminentemente participativo. Creemos que es una idea muy interesante, con muchas posibilidades, pero debemos insistir en que toda su capacidad, todo su potencial, no será aprovechado sin la colaboración y participación de los propios profesionales de la orientación y el asesoramiento vocacional. De todos ellos, de todos nosotros, va a depender la mayor o menor consecución de los ambiciosos objetivos planteados.

A continuación desarrollamos la valoración de la herramienta, mediante una tabla adaptada de la ficha propuesta por Marquès (2001) para la evaluación de espacios web de interés educativo, de acuerdo con una serie de items que consideramos deben ser tenidos en cuenta en este tipo de instrumentos. Entendemos que esta ficha puede ser utilizada por cualquier orientador para realizar su valoración del WIKI y también puede servir como modelo para evaluar otros espacios web de similares características o que puedan ser de interés para el asesoramiento vocacional y la orientación en general.

En esta valoración han intervenido ocho estudiantes de doctorado que realizaban el curso sobre "Departamento de Orientación en Secundaria" en la Universitat Jaume I de Castellón, cuatro estudiantes que han llevado a cabo el Prácticum en el Departamento de Orientación de un centro de secundaria y cuarenta profesionales, que han participado en el "Seminario para orientadores y orientadoras de educación secundaria: herramientas informáticas para el asesoramiento vocacional", organizado por la Unitat de Suport Educatiu (USE) de la Universitat Jaume I de Castellón.

\begin{tabular}{|c|c|c|c|c|c|}
\hline \multicolumn{6}{|c|}{ ASPECTOS FUNCIONALES. UTILIDAD } \\
\hline & Muy alta & Alta & Correcta & Baja & Muy baja \\
\hline $\begin{array}{l}\text { Relevancia, interés de los contenidos y servicios que ofrece } \\
\text { para el asesor vocacional }\end{array}$ & $\mathrm{X}$ & & & & \\
\hline Facilidad de uso & & $\mathrm{X}$ & & & \\
\hline $\begin{array}{l}\text { Claridad (objetivos, contenidos y procesos claros; lenguaje } \\
\text { que facilita la comprensión, ...) }\end{array}$ & & $\mathrm{X}$ & & & \\
\hline $\begin{array}{l}\text { Versatilidad (funcionalidad en diferentes contextos —aula de } \\
\text { informática, clase con un único ordenador, uso doméstico...- } \\
\text { y posibilidad de integrarse fácilmente con otros medios o } \\
\text { actividades previas o posteriores al propio uso del instrumento) }\end{array}$ & $X$ & & & & \\
\hline Viabilidad (realista/de posible aplicación) & $\mathrm{X}$ & & & & \\
\hline Eficacia (conduce a los resultados previstos) & & $\mathrm{X}$ & & & \\
\hline Eficiencia (balance coste-beneficio) & $x$ & & & & \\
\hline Interacción y canales de comunicación bidireccional & $\mathrm{X}$ & & & & \\
\hline Servicios de apoyo on-line (ayuda, tutoriales, ...) & & $\mathrm{X}$ & & & \\
\hline
\end{tabular}




\begin{tabular}{|c|c|c|c|c|c|}
\hline $\begin{array}{l}\text { Evaluación y evaluabilidad (autocontrol del trabajo realizado } \\
\text { por parte del propio usuario y posibilidad de evaluación y } \\
\text { mejora del instrumento) }\end{array}$ & & $\mathrm{X}$ & & & \\
\hline Funcionalidad de la documentación & & $\mathrm{X}$ & & & \\
\hline Ausencia o poca presencia de publicidad & $x$ & & & & \\
\hline \multicolumn{6}{|c|}{ ASPECTOS TÉCNICOS Y ESTÉTICOS } \\
\hline & Muy alta & Alta & Correcta & Baja & Muy baja \\
\hline Entorno audiovisual: presentación, pantallas, letra... & & $\mathrm{X}$ & & & \\
\hline Calidad y estructuración de los contenidos & & $x$ & & & \\
\hline Estructura y navegación por las actividades & $x$ & & & & \\
\hline Hipertextos descriptivos y actualizados & $X$ & & & & \\
\hline Ejecución fiable, velocidad de acceso adecuada & $\mathrm{X}$ & & & & \\
\hline Originalidad y uso de tecnología avanzada & $x$ & & & & \\
\hline \multicolumn{6}{|l|}{ ASPECTOS PSICOLÓGICOS } \\
\hline & Muy alta & Alta & Correcta & Baja & Muy baja \\
\hline Capacidad de motivación, atractivo, interés & & $x$ & & & \\
\hline $\begin{array}{l}\text { Adecuación a los destinatarios de los contenidos, } \\
\text { actividades... }\end{array}$ & $\mathrm{X}$ & & & & \\
\hline Satisfacción del usuario & & $X$ & & & \\
\hline \multicolumn{6}{|c|}{ VALORACIÓN GLOBAL DEL WIKI } \\
\hline & Muy alta & Alta & Correcta & Baja & Muy baja \\
\hline Funcionalidad, utilidad & $x$ & & & & \\
\hline Calidad Técnica & $X$ & & & & \\
\hline Atractivo & & $x$ & & & \\
\hline
\end{tabular}

Tanto la funcionalidad/utilidad como la calidad técnica del WIKI DE LA ORIENTACIÓN Y EL ASESORAMIENTO VOCACIONAL han recibido una valoración muy alta. Entre los aspectos funcionales, destaca la valoración que reciben los servicios ofertados, la versatilidad, viabilidad y eficiencia de la herramienta y el apartado relacionado con la interacción y los canales de comunicación bidireccional. La facilidad de uso y la claridad reciben una alta valoración en general, sin embargo en algunos casos se puntúan con un aprobado justo, a causa de una cierta complejidad observada en el modo edición.

La calidad técnica es valorada como muy alta gracias a la estructura y navegación por sus diferentes apartados, el uso de los hipertextos, la fiabilidad en su ejecución y su velocidad de acceso, y la originalidad de la tecnología implementada. 
Parece desprenderse, pues, de esta valoración, que calidad técnica y funcionalidad se dan la mano para conseguir que el WIKI DE LA ORIENTACIÓN Y EL ASESORAMIENTO VOCACIONAL se convierta en el entorno abierto, flexible y participativo que pretende ser.

Como posibles mejoras, cabría revisar los niveles de complejidad y algunas de las dificultades encontradas en el modo edición, mejora que redundará, sin duda, en la mejora de la motivación y la satisfacción de los usuarios, de manera que los aspectos psicológicos que, en la actual valoración, reciben una nota alta, puedan alcanzar la máxima valoración.

\section{Referencias bibliográficas}

Castells, M. (2000). La era de la información. La sociedad red. Alianza, Madrid.

Castillo, J. (1999). Aplicación de herramientas groupware a través de Internet: BSCW. Su utilidad en las Comunidades Virtuales de Usuarios. Documento electrónico accesible en http://www.rediris. es/cvu/publ/bscw $99 . h t m l$

Coma del Corral, M. (1998). Uni-Net: red de recursos telemáticos integrados para comunidades virtuales de usuarios. VII Congreso nacional de informática médica, Pamplona.

Gisbert, M. y Feliu, V. (2000). “¿Para qué sirven las listas de distribución?”. En Pérez, R. y otros (coords), Redes, multimedia y diseño de entornos virtuales. Oviedo: Universidad de Oviedo.

Harasim, L. Hiltz, S., Teles, L. y Turoff, M. (1995). Learning Networks. Cambridge, Massachusetts: The MIT Press.

Hills, M. (1997). Intranet para groupware. Madrid: Anaya multimedia.

Marquès, P. (1999). Criterios para la clasificación y evaluación de espacios web de interés educativo. Documento web disponible en http://dewey.uab.es/pmarques

Marquès, P. (2001). Ficha para la evaluación de materiales multimedia. Documento web disponible en http://dewey.uab.es/pmarques

Miller, D. (1999). "Establiushing a community information network". En Pantry, S. (ed), Building community information networks. Strategies and experiences. London: Library Association Pub.

Pazos, Mª, Pérez, A. y Salinas, J. (2001). COMUNIDADES VIRTUALES: De las listas de discusión a las comunidades de aprendizaje. Comunicación presentada en EDUTEC 2001. Congreso Internacional de Tecnología, Educación y Desarrollo Sostenible. Murcia, 17-19 septiembre.

Salinas, J. (1998). Redes y desarrollo profesional del docente: Entre el dato serendipiti y el foro de trabajo colaborativo. http://www.uib.es/depart/gte/docente.html

Salinas, J. (2000). “El aprendizaje colaborativo con los nuevos canales de comunicación”. En Cabero, J., Salinas, J. Duarte, A y Domingo, J., Nuevas Tecnologías aplicadas a la Educación. Madrid: Síntesis.

Sanz, J. (2005). Nuevas Tecnologías Aplicadas al Asesoramiento Vocacional. Diseño de Software y Servicios de Apoyo Telemático. Tesis doctoral. Universitat Jaume I de Castellón.

Fecha de recepción: 08-06-06

Fecha de revisión: 26-09-07

Fecha de aceptación: 07-11-07 